Published in Cryogenics 18, 201-208 (1978).

\title{
An Apparatus for Preparing Isotopically Pure ${ }^{4} \mathrm{He}$
}

\author{
P. V. E. McClintock \\ Department of Physics, \\ University of Lancaster, \\ Lancaster LAl 4YB, UK.
}

The helium extracted from gas wells consists predominantly of ${ }^{4} \mathrm{He}$ but it also contains traces $^{1}$ (a few parts in $10^{7}$ ) of the other stable isotope, ${ }^{3} \mathrm{He}$. For many purposes, the presence of the ${ }^{3} \mathrm{He}$ may be neglected. Commercial ${ }^{4} \mathrm{He}$, widely used for a variety of cryogenic and other technological applications, usually consists of the two isotopes in their natural relative abundances.

There are certain other circumstances, however, where the presence of even this very small proportion of isotopic impurity can be of great significance, and it then becomes essential for the ${ }^{4} \mathrm{He}$ to be purified. Examples include: studies of phonon scattering in solid helium $^{2}$; measurements of the surface properties of the liquid at very low temperatures ${ }^{3}$, investigations of the ideal breakdown of superfluidity ${ }^{4,5}$ using negative ions at millikelvin temperatures ${ }^{6,7}$; the use of ${ }^{4} \mathrm{He}$ gas as a coolant in high temperature nuclear reactors (HTRs) ${ }^{8}$; and the recently proposed construction of a superthermal source of ultra-cold neutron (UCN) gas, using superfluid ${ }^{4} \mathrm{He}$ as the internal medium ${ }^{9}$. The isotopic purity required for the latter application is perhaps the most exacting of all: in order that the average neutron lifetime inside the containing bottle should be limited by $\beta$-decay, rather than through absorption by ${ }^{3} \mathrm{He}$ atoms, it is necessary that the ${ }^{4} \mathrm{He} /{ }^{3} \mathrm{He}$ ratio $\left(=R_{43}\right)$ should be substantially in excess of $2 \times 10^{10}$, i.e. about four orders of magnitude greater than that of natural helium. The purity criterion for the millikelvin ion experiments is almost as stringent, it being necessary for a negative ion to be able to travel about $10 \mathrm{~cm}$ through superfluid ${ }^{4} \mathrm{He}$ without encountering any ${ }^{3} \mathrm{He}$ atoms: the latter have a tendency to 
condense $^{10}$ on the outside of the ion, leading to the nucleation of charged vortex rings ${ }^{6,11}$ and effectively suppressing the roton creation mechanism which is under investigation. To prevent this condensation phenomenon from occurring, it is necessary to ensure that $R_{43}>10^{10}$.

The techniques which.have been developed for isotopically purifying ${ }^{4} \mathrm{He}$ include: "filtration" of natural He II through a superleak ${ }^{12,13}$; differential distillation at temperatures below the lambda point ${ }^{14}$; and heat flush ${ }^{15}$ : typical purities attained are displayed in Table 1. It will be noted that, with one likely exception ${ }^{15}$, the product could in no case have been pure enough for either the neutron containment project or the proposed millikelvin

ion experiments ${ }^{7}$. The purposes of the present work have been, first, to prepare ${ }^{4} \mathrm{He}$ of greater isotopic purity than has hitherto been attained, sufficient for the latter investigations and, secondly, to place on $R_{43}$ an experimental lower bound much larger than that achievable using straightforward mass spectrometry for analysing the product. In short, the aim was to prepare in liquid litre quantities some ${ }^{4} \mathrm{He}$ which was demonstrably pure enough to be used for any existing or projected application.

As described below, the purification was carried out by means of the heat flush technique. To establish limits on the isotopic purity level which had been achieved, a secondary heat flush was used to drive any ${ }^{3} \mathrm{He}$ atoms into a small subsidiary chamber, from which a concentrated sample could then be taken for subsequent analysis by mass spectrometry. A brief preliminary report ${ }^{16}$ on these experiments has already been published.

\section{Isotopic purification by means of heat flush}

The heat flush technique relies on the fact that ${ }^{3} \mathrm{He}$ atoms in He II form part of the normal fluid component ${ }^{17}$. Thus, in thermal counterflow, where normal fluid travels away from a source of heat and superfluid simultaneously moves towards it, any ${ }^{3} \mathrm{He}$ atoms will tend to congregate at the colder end of the apparatus. Early work on helium isotope separation using a heat flush technique ${ }^{18,19}$ was, for historical reasons, directed towards the production of ${ }^{3} \mathrm{He}$, no particular effort being made to isolate or to measure the purity of the ${ }^{4} \mathrm{He}$ which was being produced as a byproduct. It is shown below that heat flush is, in fact, an extraordinarily powerful technique for separating the isotopes, and particularly so for preparing isotopically pure ${ }^{4} \mathrm{He}$. The concentration of ${ }^{3} \mathrm{He}$ which can be produced 
is curtailed by the non-superfluidity of ${ }^{3} \mathrm{He}-{ }^{4} \mathrm{He}$ solutions ${ }^{20}$ containing more than about $67 \%$ of ${ }^{3} \mathrm{He}$; but no such intrinsic limitation exists to govern the purity of the ${ }^{4} \mathrm{He}$ which can be prepared by this method.

The basis of ${ }^{4} \mathrm{He}$ purification by means of heat flush is shown diagrammatically in Fig. 1. The normal fluid component, flowing away from a heater, will tend to carry with it any ${ }^{3} \mathrm{He}$ atoms which initially were within the flushing tube and to prevent others from entering. The isotopically pure superfluid component can then be drawn off as indicated in the opposite direction. The particular function of the superleak is to define the direction in which most of the normal fluid flows away from the heater; it is not intended to act as a purifier in its own right and its quality (as a barrier to normal fluid) is not, therefore, a critical factor on which the success of the technique will depend. The degree of purification achieved will depend on the extent to which ${ }^{3} \mathrm{He}$ atoms are able to diffuse against the counterstream of normal fluid; and this in turn is likely to depend on factors such as the normal fluid velocity, the coefficient of diffusion for ${ }^{3} \mathrm{He}$ atoms in He II, and the length of the flushing tube.

To analyse the situation quantitatively, we treat the general problem of diffusion within a moving medium. For simplicity, however, we consider only the steady state situation and we ignore the transient effects which will occur just after the initial conditions are established: a steady state should be approached within a few normal fluid transit times after the heater is energized; and, for our experimental conditions, the transit time from heater to main bath is at most a few seconds. We will assume that, viewed from a frame of reference fixed in the normal fluid, movement of the ${ }^{3} \mathrm{He}$ atoms by thermal diffusion is unaffected either by the magnitude of the relative velocity between the normal fluid and the laboratory, or by that between the normal fluid and the superfluid.

In the laboratory frame of reference the flux $\dot{q}$ of ${ }^{3} \mathrm{He}$ atoms is given generally by

$$
\dot{q}=-D \operatorname{grad} u+u \boldsymbol{v}
$$

where $D$ is their coefficient of diffusion at the relevant temperature, $u$ is the number density of ${ }^{3} \mathrm{He}$ atoms and $\boldsymbol{v}$ is the velocity of the moving medium (the normal fluid component). We now consider an arbitrary volume $V$ bounded by a surface $S$. In the steady state, conservation of matter requires that the net rate of flow across the surface 
must be zero so that, using (1), we obtain

$$
\int_{S}(-D \operatorname{grad} u+u \boldsymbol{v}) \cdot \boldsymbol{d} s=0
$$

which, on applying Gauss's theorem, becomes

$$
\int_{V} \operatorname{div}(D \operatorname{grad} u-u \boldsymbol{v}) \cdot \boldsymbol{d} s=0
$$

Remembering that the volume $V$ is arbitrary, and using a standard vector identity, this yields

$$
D \nabla^{2} u-u \operatorname{div} \boldsymbol{v}-\boldsymbol{v} \cdot \operatorname{grad} u=0
$$

To a good approximation, the temperature and hence the normal fluid density remain constant throughout a bath of He II, so that the equation of continuity for the normal fluid implies $\operatorname{div} \boldsymbol{v} \simeq 0$.

We therefore obtain finally

$$
D \nabla^{2} u-\boldsymbol{v} \cdot \operatorname{grad} u=0
$$

We note that this differs from Laplace's equation, which describes steady state diffusion in a stationary medium, by virtue of the translational term $\boldsymbol{v} \cdot \operatorname{grad} u$ but that, as required, the expression reduces to Laplace's equation in the limit where $v$ tends to zero.

If the coordinate frame of reference is orientated such that $v_{x}=-v_{n}, v_{y}=v_{z}=0$, (3) becomes

$$
\nabla^{2} u+\frac{v_{n}}{D} \frac{\partial u}{\partial x}=0
$$

a form which, in allusion to an obvious analogy, is sometimes known as the equation of gas attack. For diffusion within a long tube of constant cross section, (4) reduces to the one-dimensional form

$$
\frac{d^{2} u}{d x^{2}}+\frac{v_{n}}{D} \frac{d u}{d x}=0
$$

Solving subject to the boundary conditions appropriate to a semi-infinite tube open at one end to the bath: $u=d u / d x=0$ at $x=\infty ; u=u_{0}$ at $x=0$; (5) yields 


$$
u=u_{0} \exp \left(-v_{n} x / D\right)
$$

This simple expression determines the extent to which ${ }^{3} \mathrm{He}$ atoms are able to diffuse against a wind of counterflowing normal fluid component within the flushing tube of an isotopic purification cryostat arranged as shown in Fig. 1. Substitution of typical numerical values demonstrates immediately the remarkable efficacy of heat flush as a means of removing ${ }^{3} \mathrm{He}$ isotopic impurities from He II: with $v_{n}=2 \mathrm{~cm} \mathrm{~s}^{-1}, x=5 \mathrm{~cm}, D=2 \times 10^{-3} \mathrm{~cm}^{2} \mathrm{~s}^{-1}$ (for $T=1.4 \mathrm{~K})^{21}$, we find $u / u_{0}=3.4 \times 10^{-2172}$; alternatively, using the same values of $v_{n}$ and $D$ and assuming that the flushing tube is open at $x=0$ to a He II bath of natural isotopic purity, we may calculate that the liquid will on average be entirely devoid of ${ }^{3} \mathrm{He}$ atoms (i.e. less than $1{ }^{3} \mathrm{He}$ atom $\mathrm{cm}^{-3}$ ) for $x \geq 0.4 \mathrm{~mm}$. We note that this very short distance within which the average ${ }^{3} \mathrm{He}$ concentration falls to zero is consistent with our implicit assumption, above, that a flushing tube of a few $\mathrm{cm}$ length could without appreciable error be regarded as being semi-infinite in extent.

It may be concluded that, at least in principle, the use of heat flush constitutes a technique capable of achieving total removal of the ${ }^{3} \mathrm{He}$ isotopic impurities from a given sample of natural ${ }^{4} \mathrm{He}$.

\section{Measurement of the isotopic purity of the product}

Although the above considerations indicate that the level of ${ }^{3} \mathrm{He}$ impurities in the ${ }^{4} \mathrm{He}$ product is likely to be undetectable, it is obviously desirable to test the product in the most sensitive manner possible and, preferably, to be able to place on its $R_{43}$ ratio an experimentally determined lower bound which is substantially greater than the smallest value of $R_{43}$ for which the project of immediate concern can be expected to succeed; for example, $R_{43} \gg 2 \times 10^{10}$ in the case of neutron containment. It is unfortunate that mass spectrometry, which appears to be the most sensitive analytical technique currently available, has an ultimate sensitivity which falls far short of this aim, being restricted to measurements of $R_{43}<2.5 \times 10^{9}$. It is to get round this limitation that the present isotopic purification cryostat incorporates a secondary heat flush to concentrate any ${ }^{3} \mathrm{He}$ atoms, which might by some unknown means have found their way into the product, prior to taking the sample for analysis. 
A problem in designing the arrangements for this secondary flush lay in maintaining reasonable values of $v_{n}$ throughout the whole volume of purified ${ }^{4} \mathrm{He}$. It was intended to collect the ${ }^{3} \mathrm{He}$ atoms (if any) in a small pot separated from the main pot of purified ${ }^{4} \mathrm{He}$ by a needle valve, which could be shut as soon as the flush had been completed; and it was clearly inconvenient to make the orifice of this needle valve larger than of a few $\mathrm{mm}$ id. Thus, if all the heat input to operate the secondary flush had had ultimately to pass through the needle valve, it would have been necessary to limit its magnitude severely in order to avoid cavitation and boiling ${ }^{22}$ within the orifice; and $v_{n}$ would have taken a correspondingly low value throughout much of the main pot, which of necessity had an inner diameter of several $\mathrm{cm}$. One consequence would have been a serious danger that back-diffusion would lead to incomplete collection of ${ }^{3} \mathrm{He}$ atoms in the sample pot.

Accordingly, it was necessary to operate the heater at a higher level and, at the same time, to separate most of the thermal fraction of the normal fluid from the ${ }^{3} \mathrm{He}$ before the latter passed through the needle valve. This was accomplished by means of thermal exchange with the walls of a small-angled copper cone.

The utility of the conical heat exchanger, shown diagramatically in Fig. 2, rests on the fact that the average axial normal fluid velocity in such a device is almost independent of position along the axis. To show this, we note that, to a good approximation, there will be no temperature gradients in the He II or in the copper walls of the cone. There will, however, be the usual Kapitza discontinuity $\Delta T$ between the temperature of the He II and that of the walls. The rate at which heat passes across any given radial cross section in the cone will therefore be proportional to the area of copper wall lying beyond it. Thus the ratio $\phi$ of the thermal flux at $y=Y$ to that entering the cone at $y=0$ is

$$
\phi=\frac{A_{C Y} / A_{B Y}}{A_{C 0} / A_{B 0}}
$$

where $A_{C O}$ and $A_{B O}$ are respectively the areas of the curved wall and of the base for the whole cone, and $A_{C Y}$ and $A_{B Y}$ those of the cone within $Y \leq y \leq L$. The area of the curved wall of a cone of length $h$ and base of radius $r$ is $\pi r\left(r^{2}+h^{2}\right)^{1 / 2}$, so we can write

$$
\phi=\frac{\pi r_{Y}\left[r_{Y}^{2}+(L-Y)^{2}\right]^{1 / 2} / \pi r_{Y}^{2}}{\pi r_{0}\left(r_{0}^{2}+L^{2}\right)^{1 / 2} / \pi r_{0}^{2}}
$$


where $r_{0}$ and $r_{Y}$ are the radii of the cone at $y=0$ and $y=Y$ respectively, and $L$ is its length. Thus

$$
\phi=\left\{\frac{1+\left[(L-Y) / r_{Y}\right]^{2}}{1+\left(L / r_{0}\right)^{2}}\right\}^{1 / 2}
$$

Now, for a cone,

$$
r_{Y}=r_{0}(L-Y) / L
$$

Numerator and denominator are therefore equal; and $\phi=1$. Because the average thermal flux $\dot{Q}$ therefore remains constant along the length of the cone, so also will the normal fluid velocity $v_{n}$, which is given by ${ }^{17}$

$$
v_{n}=\dot{Q} / \rho S T
$$

where $\rho, S$ and $T$ are respectively the density, entropy and temperature of the He II inside the cone.

It was hoped, therefore, that all ${ }^{3} \mathrm{He}$ atoms carried into the cone by the flow of normal fluid would be swept along at a constant speed right up to its apex at $y=L$, where they would then pass through the needle valve and be collected in the small sample chamber (not shown in Fig. 2); but that most of the normal fluid entering at the base of the cone would be able to flow as heat into the copper walls before reaching the needle valve. The device could not, of course, reasonably be expected to operate efficiently under laminar flow conditions because the component of $v_{n}$ perpendicular to the (inevitably) microscopically rough walls would be liable to hold ${ }^{3} \mathrm{He}$ atoms in position "plated" onto the copper surface, preventing most of them from leaving the cone: rather, it was intended for operation using values of $v_{n}$ large enough to produce highly turbulent normal fluid flow within the cone. For the same reason, it seemed advisable to use a cone of as small an angle as was convenient, so that, for any given value of $v_{n}$, the component perpendicular to the walls would be minimised.

The likely efficiency of the concentrator was hard to foresee since no similar advice had been reported previously, and because the (all important) microscopic normal fluid flow patterns very close to the walls would have been difficult to calculate with any confidence. 
For this reason it was felt preferable to adopt an experimental approach and to determine the efficacy of the secondary heat flush by means of a calibration experiment: by placing in the main pot a sample of ${ }^{4} \mathrm{He}$ with a relatively high ${ }^{3} \mathrm{He}$ content, operating the cone concentrator, and subsequently comparing the isotopic purities of ${ }^{4} \mathrm{He}$ taken from the sample chamber and from the main pot.

To approach the ideal situation assumed in the above model, with a constant temperature throughout the walls of the cone, by using the arrangement of Fig. 2 would have been inconvenient in practice because extremely thick walls of very high purity copper would have been required. Instead, it was felt preferable to use relatively thin copper walls cooled on their outer surface by a pumped bath of (impure) He II.

\section{The isotopic separation cryostat}

The lower part of the cryostat insert which was used in practice is shown diagrammatically in Fig. 3. The whole dipped into a bath of He II of natural isotopic purity in a metal dewar of id $164 \mathrm{~mm}$. The bath could be pumped by a 3,500 litre/min vacuum pump ${ }^{23}$ connected to the dewar via $15 \mathrm{~m}$ of rigid $100 \mathrm{~mm}$ id, and $2 \mathrm{~m}$ of flexible $80 \mathrm{~mm}$ id, pumping line. The vacuum space which surrounded the main pure helium pot $\mathrm{d}$ could be pumped via tube $\mathrm{m}$, which also enabled helium exchange gas to be admitted temporarily to assist in cooling the apparatus down from room temperature. The salient design features of the cryostat are most conveniently described by considering the sequence of operations required for running it.

Initially, all the internal liquid helium spaces were under vacuum, and the three needle valves $\mathrm{V}_{1}, \mathrm{~V}_{2}$ and $\mathrm{V}_{3}$ were a11 closed. Once the main bath a had been filled and pumped down to below the lambda point, the heater $\mathrm{H}_{1}$ was energised in order to provide the primary heat flush within the main flushing tube b which was $9.6 \mathrm{~mm}$ id, and $12 \mathrm{~cm}$ long. The needle valve $V_{1}$ was then cautiously opened, admitting isotopically pure He II via the superleak c to the back of $V_{2}$. The function of $V_{1}$ was to protect the superleak from contamination by helium of natural isotopic purity while the main bath was being filled and pumped down, a process which is believed to have limited the ultimate purity of the

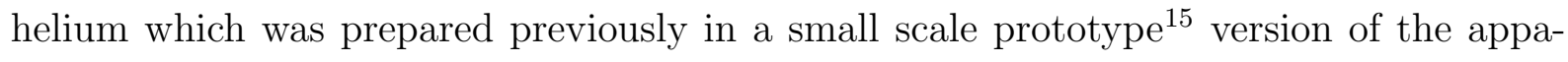
ratus. (The tubes $\mathrm{n}$ and o enabled the superleak to be evacuated at room temperature, 
and also allowed trapped pockets of liquid to evaporate harmlessly when the apparatus warmed up at the end of the experiment.) The valve $\mathrm{V}_{2}$ was then opened, and the main pot $\mathrm{d}$ for the purified ${ }^{4} \mathrm{He}$ allowed to fill.

It was, of course, essential to ensure that d had filled completely since, otherwise, the cone concentrator would not have been able to fulfil its proper function: the hollow brass fullness indicator e constituted a vital part of the system. This operated by measuring the temperature difference $\Delta T$ between thermometers $T_{2}$ and $T_{3}$ as a function of the power applied to the heater $\mathrm{H}_{3}$. With the pot evacuated, a very small heat input $\dot{Q}_{3}$ produced a large $\Delta T$; after $\mathrm{V}_{2}$ had been opened and the pot was filling, film flow caused $\Delta T$ to remain essentially equal to zero for a range of small $\dot{Q}_{3}$ values; but as soon as the liquid has filled the inside of the indica.tor, $\Delta T$ remained equal to zero for a very wide range of $\dot{Q}_{3}$. It was therefore possible to obtain separate indications showing: first that $\mathrm{V}_{1}$, c and $\mathrm{V}_{2}$ were all open, and that the pot was filling; and, subsequently, that the pot was completely full, so that it was appropriate for the secondary flush to commence.

While the pot $d$ was filling, it was vital to ensure that the flushing tube b was fully immersed in He II. This was accomplished by means of a movable liquid level indicator $\mathrm{T}_{4}$ which was capable ${ }^{24}$ of locating the position of the He II surface in the main bath to $\pm 1 \mathrm{~mm}$. It was therefore possible to monitor very accurately the rate at which the level was falling in the dewar. When the level had stopped falling, and e confirmed that the pot was full, it was assumed that the liquid extended up to the top of the cone. The valve $\mathrm{V}_{3}$ was then opened to allow the level to pass beyond the small sample pot $\mathrm{g}$ and, after a short pause, $\mathrm{V}_{2}$ was closed.

Heat was then supplied to the outer copper surface of d, using the heaters represented by $\mathrm{H}_{2}$. The relatively poor thermal conductivity of the brass tube i which formed the main support between $\mathrm{d}$ and the vacuum can $\mathrm{j}$ ensured that almost all of this heat entered the helium in $\mathrm{d}$, producing a flow of normal fluid into the bottom of the cone. Because $\mathrm{d}$ was constructed entirely of copper, all of its surfaces could in effect be regarded as exuding normal fluid, thus ensuring that no ${ }^{3} \mathrm{He}$ atoms could be left behind in crevices or other small "backwaters", but would all eventually be swept towards and into the cone f.

To discourage ${ }^{3} \mathrm{He}$ atoms from becoming plated onto the inner walls of the cone (see above) the angle of the cone was made as small as possible. To accomplish this without 
lengthening the apparatus to an unacceptable extent, the cone was effectively folded into the pot: by starting it from a position near the bottom of $d$, it was possible to make it a great deal longer, with a half angle of only $0.80^{\circ}$ compared to the $2.70^{\circ}$ which would have been necessary had it entered $\mathrm{d}$ at the top. It may be noted that the non-negligible area of (copper) surface presented by the small pot g, ignored in our analysis above of the characteristics of the cone, will cause the normal fluid to accelerate as it approaches the apex. When the secondary flush was judged complete, with any ${ }^{3} \mathrm{He}$ atoms having been swept up into $\mathrm{g}, \mathrm{V}_{3}$ was closed. The apparatus was then left to warm up towards room temperature, evaporating the product in $\mathrm{d}$, which was allowed to pass out through the exit tube $\mathrm{k}$ and to pressurise a standard gas bottle to $35 \times 10^{5} \mathrm{Nm}^{-2}$. The gas which evaporated from $\mathrm{g}$ was collected in a small sample bottle for subsequent analysis.

Some details of the method of construction are shown in Figs. 4 and 5. The brass vacuum can was bolted to its flange over an indium '0' ring by means of $186 \mathrm{~mm}$ steel bolts. The curved walls of the main copper pot were fabricated from $6 \mathrm{~mm}$ copper sheet, rolled and welded to form a cylinder. The copper end caps, made from $12 \mathrm{~mm}$ sheet, were hard-soldered into position. In order to allow access to the rather complicated joint at the bottom of the cone, the pot could be removed by running the central soft soldered joint at the top: to keep the latter safely to a reasonable length, in view of the required working pressure, it was made of as small a diameter $(30 \mathrm{~mm})$ as possible. The cone itself was also fabricated from copper sheet, this time of $1 \mathrm{~mm}$ thickness, again with a welded seam and with end fittings hard soldered into place. It was $33 \mathrm{~cm}$ in length, of $12 \mathrm{~mm}$ id at its base and of $2 \mathrm{~mm}$ id at the top. The cone was soft soldered into position at both ends so that it could easily be removed if any leaks developed. The tubes for carrying liquid helium between the superleak, needle valves and main pot were of $3 \mathrm{~mm}$ id brass. A number of sliding brass sleeves, soft soldered into place, were used as connecting pieces in order to make the whole arrangement easily demountable. The superleak itself consisted of a plug of compressed jeweller's rouge $20 \mathrm{~mm}$ in length inside a $10 \mathrm{~mm}$ id cylindrical hrass housing: rouge was used (rather than Vycor glass ${ }^{13,15}$ ) so as to produce a superleak with a relatively large volume flow rate, so that the main pot would fill quickly. The needle valves were of stainless steel, operating against brass seats and, in the cases of $V_{2}$ and $V_{3}$, were tested to $35 \times 10^{5} \mathrm{~N} \mathrm{~m}^{-2}$ working pressure. 
The shaft seals were ' 0 ' rings at room temperature. The fixed thermometers were $470 \Omega 1 / 2 \mathrm{~W}$, Speer carbon resistors glued into copper sleeves, which were then Wood's metalled to the apparatus; and the movable level indicator was based on a $47 \Omega, 1 / 8 \mathrm{~W}$, Allen-Bradley carbon resistor mounted as described previously ${ }^{24}$. The thermometers were all calibrated against the ${ }^{4} \mathrm{He}$ vapour pressure in the main dewar. The heaters were $1 / 2 \mathrm{~W}$ metal film resistors also glued into copper sleeves and Wood's metalled into position. In addition to the principal heaters illustrated in Fig. 3, subsidiary heaters were fitted on the pot filling tube immediately below $\mathrm{V}_{2}$ and on tube $\mathrm{k}$ just above the main pot $\mathrm{d}$. Their function was to ensure that, during the secondary heat flush, there was a net flow of normal fluid out of these tubes and into d, thus preventing stagnation of ${ }^{3} \mathrm{He}$ atoms which otherwise might have escaped being driven up into g and remained behind to contaminate the product. In the case of $\mathrm{k}$, a $0.3 \mathrm{~mm}$ orifice was inserted just above the heater, to make quite sure that some of the heat went down into d (and that it did not merely contribute to a flow of heat from d up k into the main He II bath, caused by the fact that, during the secondary flush, the helium in $\mathrm{d}$ is at a significantly higher temperature than that of the main bath). A similar orifice was placed in tube 1 just above the sample pot $\mathrm{g}$ in order to eliminate the possibility of a reverse heat flush from 1 towards the cone: in practice, the liquid level in 1 was often significantly above that in the main bath and the resultant heat load could, in the absence of an orifice, sometimes have been sufficient to produce a reverse flush from this tube comparable with that which otherwise would have entered g from the cone.

\section{Experimental results}

Three experimental runs have been performed: the first was to test the apparatus, to establish appropriate procedures and operating conditions, and to obtain a supply of isotopically pure ${ }^{4} \mathrm{He}$ for flushing the room temperature pipework and storage cylinder; the second was the main purification run to prepare the final product, amounting to the equivalent of about 2.4 litres of isotopically pure liquid ${ }^{4} \mathrm{He}$; and the third was to calibrate the efficacy of the cone concentrator.

The initial charge of helium amounted to about 21 litres of liquid at $4.2 \mathrm{~K}$, of which 19 litres were situated above the vacuum can flange. A sample of the gas (A) was collected 
for subsequent analysis. After the dewar had been pumped down, over a period of about 2 hours, it was found that some 10 litres of liquid at $1.1 \mathrm{~K}$ remained above the flange. The equilibrium temperature of the main bath as a function of heat input was measured, giving the points shown as squares in Fig. 6. Using (7) to compute the corresponding normal fluid velocities within the flushing tube as a function of heat input yielded the upper curve of Fig. 7. In practice the heat input at $\mathrm{H}_{1}$ (Fig. 3) for the primary heat flush was set at $0.15 \mathrm{~W}$, giving a bath temperature of $1.24 \mathrm{~K}$ and $v_{n}=18 \mathrm{~cm} \mathrm{~s}^{-1}$ at the exit of the tube. For this temperature, it took 50 minutes for 2.4 litres of liquid to pass through the superleak into the pot, indicated by a fall of $12 \mathrm{~cm}$ in the level of the helium in the main bath. Within the flushing tube, therefore, the superfluid must have had a velocity component of $1.6 \mathrm{~cm} \mathrm{~s}^{-1}$ over and above the velocity arising from the thermal counterflow: under these conditions, one would expect ${ }^{25}$ vorticity created in the counterflow to have been moving towards the main bath and there would have been no tendency for ${ }^{3} \mathrm{He}$ atoms, entrained by the tangle of vortex lines, to be carried against the normal fluid flow towards the superleak. We note that, for large heat fluxes, $v_{n}$ would actually have decreased slightly along the tube towards the superleak because of the temperature gradient ${ }^{26}$ in the liquid.

The characteristics for the secondary flush, showing the temperature of the helium in $\mathrm{d}$ as a function of the heat input to $\mathrm{H}_{2}$ (Fig. 3) are shown by the circled points of Fig. 6. These fall above those at equivalent powers for the main bath mainly because of the Kapitza boundary resistance across the cone, but also because of the temperature gradient within the helium itself ${ }^{26}$. Consequently, as shown by the lower curve of Fig. 7, the normal fluid velocities at the bottom of the cone are correspondingly smaller than those achieved at the end of the flushing tube, with $v_{n}$ passing through a maximum when about $0.4 \mathrm{~W}$ is applied to the heater. The secondary flush was operated at $0.24 \mathrm{~W}$, giving a pot temperature of $1.33 \mathrm{~K}$ and a $v_{n}$ into the cone of $11 \mathrm{~cm} \mathrm{~s}^{-1}$ for four hours: under these conditions, there should have been a complete clearance of all ${ }^{3} \mathrm{He}$ isotopic and other impurities from the pot into the cone, every ten minutes. Finally, the heat input was increased to $1 \mathrm{~W}$, transiently giving rise to very much higher values of $v_{n}$ in the cone, but with the system eventually settling at a pot temperature of $1.64 \mathrm{~K}$ and $v_{n}=10$ $\mathrm{cm} \mathrm{s}^{-1}$; this situation was maintained for a further period of $1 \mathrm{~h}$, at the end of which $\mathrm{V}_{3}$ 
was closed to isolate any ${ }^{3} \mathrm{He}$ atoms in the small pot g. As the apparatus warmed up to room temperature, samples of gas were collected from the small pot (B) and from the product evaporating from the main pot $(\mathrm{C})$ for subsequent analysis.

The third experimental run, to calibrate the cone concentrator, was carried out in the same manner as the production run except that, before the main pot d had been filled, some helium doped with approximately $1.5 \mathrm{~cm}^{3}$ of ${ }^{3} \mathrm{He}$ (at standard temperature and pressure) was admitted to $\mathrm{d}$ via tube $\mathrm{n}$. The needle valve $\mathrm{V}_{2}$ was closed, and the secondary flush was then performed under what were, as far as possible, identical experimental conditions and using identical procedures to those which applied during the production run. Again, samples of gas were collected from the small pot (D) and from the large pot $(\mathrm{E})$.

All samples (A-E) were passed over charcoal in traps at liquid nitrogen temperature to eliminate traces of water vapour which might have been picked up from the room temperature pipework. This was to prevent subsequent formation of $\mathrm{HD}^{+}$ions which otherwise would have been liable to mask low levels of ${ }^{3} \mathrm{He}$ during analysis by mass spectrometry. The sample bottles themselves had been pumped for several days beforehand via the same traps. The results of the analyses are shown in Table 2. It will be noted that, on the production run, no ${ }^{3} \mathrm{He}$ could be detected in either the product or in the sample from the concentrator; and that on the calibration run, all of the ${ }^{3} \mathrm{He}$ admitted to the main pot was flushed up into the sample pot, to within the sensitivity limits $\left(R_{43}=2.5 \times 10^{9}\right)$ of the mass spectrometer used for the analyses.

From these results we may conclude that the helium collected during the production run, prior to the secondary heat flush, must have been purer than the minimum detectable level by at least a factor equal to the volume ratio of the two pots (490, allowing for the volume of liquid in the tube above the small pot), or $R_{43} \geq 1.2 \times 10^{12}$. It is reasonable to assume that the secondary flush produced the same concentration factor on the production and calibration runs: a factor $\geq 7.8 \times 10^{5}$ between the purities within the two pots after the secondary flush had been completed, or $R_{43} \geq 2 \times 10^{15}$ for the final product. 


\section{Conclusion}

The measured isotopic purity of the ${ }^{4} \mathrm{He}$ produced by the cryostat appears to be more than adequate for all applications currently in view. Indeed the same may also be said of the helium in the main pot even prior to the secondary flush, which suggests that future isotopic purification cryostats can reasonably omit altogether any provision for a secondary heat flush, thus very greatly simplifying their design and construction. There would appear to be little difficulty in building such an apparatus on an industrial scale, capable of producing pure ${ }^{4} \mathrm{He}$ in very much larger quantities than the 2,000 litres (at standard temperature and pressure) available per cycle from the present machine.

It must be emphasised that the measured purities for the product are in both cases lower limits on $R_{43}$ : the analyses are in fact fully consistent with our expectation (see above) that no ${ }^{3} \mathrm{He}$ at all will be able to enter the product by diffusing against the primary heat flush. It seems that any ${ }^{3} \mathrm{He}$ which may exist in the product, below the limits of detection, is likely to have originated from the atmosphere: air contains about $5.3 \times 10^{-4 \%}$ of helium ${ }^{1}$ and, of this, about $1.2 \times 10^{-4 \%}$ is ${ }^{3} \mathrm{He}$. Of course, any ${ }^{3} \mathrm{He}$ initially present in the large pot would eventually have been removed by the secondary heat flush; but atmospheric ${ }^{3} \mathrm{He}$ initially present in the room temperature product storage bottle cannot so easily be ignored. If, for example, the bottle had contained air at a pressure of 133.3 Nm-2, this would have produced a final $R_{43} \simeq 5 \times 10^{15}$ (even if $R_{43}$ had been infinite for the helium evaporating from the large pot). In fact, however, this bottle had been alternately pumped and flushed several times with isotopically purified ${ }^{4} \mathrm{He}$ from the first run, so that the $R_{43}$ for the product should have been several orders of magnitude higher than our measured lower bound.

Finally, it is of interest to speculate on the absolute purity of the helium. Although no effort was made to prepare atomically clean surfaces, there is reason to believe that the liquid in the large pot at the end of the secondary heat flush may have been of quite extraordinary purity. The inside surfaces of the copper pot will certainly have been generously coated with a rich variety of contaminants: it is well known that any surface exposed to the atmosphere rapidly acquires deposits of, for example, hydrocarbons, water, sulphur and chlorine. There would in addition have been an oxide layer, traces of heavy metals introduced during machining, and residues left by the fluxes used for 
welding, brazing and soft soldering. The vapour pressures of these materials are, however, negligibly small at liquid helium temperatures, so that the pot would have cryopumped itself on cooling down. Most of the contaminants may be regarded as having formed, in effect, part of the containing walls for the liquid helium. The possible exceptions are, of course, atmospheric helium (discussed above) and hydrogen. The vapour pressure of hydrogen falls rapidly with temperature: although it remains as high as $1.33 \times 10^{-5} \mathrm{Nm}^{-2}$ at $4.2 \mathrm{~K}$, the measured data ${ }^{27}$ may be extrapolated ${ }^{28}$ to about $1.33 \times 10^{-15} \mathrm{Nm}^{-2}$ at the lambda temperature, and to less than $1.33 \times 10^{-28} \mathrm{Nm}^{-2}$ at the temperature where the primary heat flush was conducted. This suggests that there would in fact have been no gaseous hydrogen in the pot just before $\mathrm{V}_{2}$ was opened. The solubility of hydrogen, and of all other materials, in helium is, in any case, believed to be zero (although colloidal suspensions are known to occur), so that it seems reasonable to conclude that the absolute purity of the helium was unaffected by the relatively dirty pot in which it was collected. It might perhaps be argued that the swirling action of the liquid entering the pot could have loosened small agglomerates of contaminant from the walls, which might conceivably have then formed a suspension. The secondary heat flush would, however, have swept any suspended material, as well as ${ }^{3} \mathrm{He}$ and all other foreign atoms, up into the small sample pot. Recalling that the flush was operated for five hours in total, under conditions such that the pot was completely purged every ten minutes and such that back diffusion, even for ${ }^{3} \mathrm{He}$ atoms, might be ignored, it seems quite probable that, at the moment when $\mathrm{V}_{3}$ was finally closed, the liquid in the pot constituted the purest macroscopic sample of any element yet to have been prepared on earth.

The assistance of the late R. J. Scott in general planning and in the initial testing of the apparatus is gratefully acknowledged: it will always remain a source of deep regret that he did not live to see the successful outcome. The work depended heavily on the technical expertise of N. Bewley, G. Caley, L. G. Marsden, I. E. Miller and A. Muirhead; and it was supported by the Science Research Council under GR/A/0388.3 and GR/A/4874.7.

We are much indebted to Mr. D. E. Emerson and the US Bureau of Mines, Amarillo, Texas for analysing our samples by mass spectrometry.

\section{References}


1. 1 Aldrich, L.T., Nier, A.O. Phys Rev 74 (1948) 1590

2. Mezhov-Deglin, L.P. Zh Eksp Tear Fiz 49 (1965) 66 (Sov Phys JETP 22 (1966) 47

3. Eckhardt, J.R., Edwards, D.O., Fatouros, P.P., Gasparini, F.M., Shen, S.Y. Phys Rev Lett 32 (1974) 706

4. Allum, D.R., McClintock, P.V.E., Phillips, A., Bowley, R.M. Phil Trans R Soc Lond A 284 (1977) 179

5. Bowley, R.M., Sheard, F.W. Phys Rev B 16 (1977) 244

6. Allum, D.R., McClintock, P.V.E. J Phys C: Solid State Phys 9 (1976) 1273

7. Sheard, F.W., Bowley, R.M. to be published

8. Hosegood, S.B., Romberg, E., Coast, G. Br Nuc Eng Soc J 5 (1966) 257, 499

9. Golub, R., Pendlebury, J.M. Phys Lett A 62 (1977) 337

10. Dahm, A.J. Phys Rev 180 (1969) 259

11. Bowley, R.M. J Phys C: Solid State Phys 9 (1976) L367

12. Mezhov-Deglin, L.P. Prib i Tekh Eksper 3 (1971) 217 (Cryogenics 12 (1972) 311)

13. Fatouros, P.P., Edwards, D.O., Gasparini, F.M., Shen, S.Y. Cryogenics 15 (1975) 147

14. Tully, P.C. "Isotopic purification of helium by differential distillation below the lambda point", US Bureau of Mines Report of Investigations 8054, Washington $(1975)$

15. Atkins, M., McClintock, P.V.E. Cryogenics 16 (1976) 733

16. Scott, R.J., McCintock, P.V.E. Phys Lett A 64 (1977) 205

17. Wilks, J. The properties of liquid and solid helium, (1967) Clarendon Press, Oxford

18. Lane, C.T., Fairbank, H.A., Aldrich, L.T., Nier, A.O. Phys Rev 73 (1948) 256

19. Soller, T., Fairbank, W.M., Crowell, A.D. Phys Rev 91 (1953) 1058

20. Ahlers, G. The physics of liquid and solid helium, I (eds Bennemann, K.H., Ketterson, J.B.) (1976) John Wiley and Sons New York, 2

21. Ptukha, T.P. Zh Eksp Teor Fiz 40 (1961) 1583 (Soviet Phys JETP 13 (1961) 1112

22. Broadwell, J.E., Liepmann, H.W. Phys of Fluids 12 (1969) 1533

23. Alley Compressors Ltd. "Geryk" vacuum pump type $12 \mathrm{X} / 63$

24. McClintock, P.V.E. Cryogenics 18 (1978) 183

25. Ashton, R.A., Northby, J.A. Phys Rev Lett 35 (1975) 1714 
26. Childers, R.K., Tough, J.T. Phys Rev B 13 (1976) 1040

27. Honig, R.E., Hook, H.O. RCA Review 21 (1960) 360

28. Bailey, C.A. Advanced Cryogenics (ed Bailey, C.A.) (1971), Plenum Press London, ch 12 


\section{Tables}

Table 1: Earlier work on the isotopic purification

\begin{tabular}{|c|c|c|}
\hline Experiment & Basis of technique & $R_{43}\left(={ }^{4} \mathrm{He} /{ }^{3} \mathrm{He}\right.$ ratio $)$ \\
\hline Mezhov-Deglin ${ }^{12}$ (1971) & Rouge superleak & $>2 \times 10^{7}$ \\
\hline Tully $^{14}(1975)$ & Distillation & $10^{9 *}$ \\
\hline Fatouros et al. ${ }^{13}(1975)$ & Vycor glass superleak & $2.5 \times 10^{9}$ \\
\hline Atkins \& McClntock ${ }^{15}$ (1976) & Heat flush & $>2.5 \times 10^{9}\left(\right.$ c. $\left.10^{12 \dagger}\right)$ \\
\hline
\end{tabular}

*typical value

†estimated value

Table 2: Isotopic analyses of samples taken from the purification cryostat

\begin{tabular}{llc}
\hline Sample & Origin of sample & $R_{43}\left(={ }^{4} \mathrm{He} /{ }^{3} \mathrm{He}\right.$ ratio $)$ \\
\hline A & starting gas & $(6.3 \pm 0.9) \times 10^{6}$ \\
B & production run, concentrated sample & $>\mathrm{mmr}^{*}$ \\
C & production run, product & $>\mathrm{mmr}$ \\
D & calibration run, concentrated sample & $(3.2 \pm 0.5) \times 10^{3}$ \\
E & calibration run, product & $>\mathrm{mmr}$ \\
\hline & ${ }^{*}$ maximum measurable ratio $\mathrm{R}_{43}=2.5 \times 10^{9}$
\end{tabular}




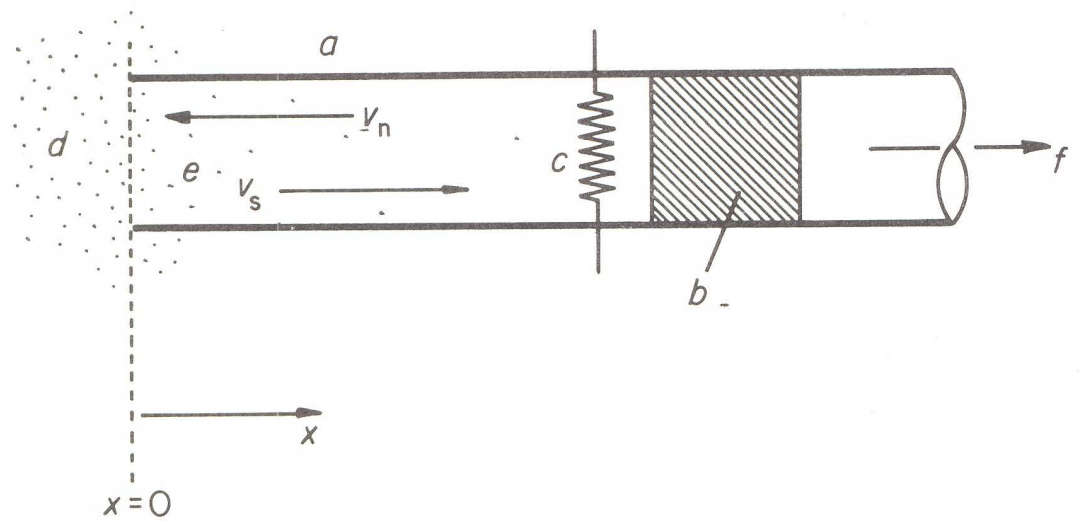

Figure 1: Isotopic purification of ${ }^{4} \mathrm{He}$ by using heat flush in He II: normal fluid flows away from the heater at velocity $v_{n}$ carrying with it the impurities (which are unaffected by the fact that the superfluid component is simultaneously flowing at velocity $v_{s}$ toward the heater) a -flushing tube, b - superleak, c - heater, d - main bath of He II of natural isotopic composition with dots to represent ${ }^{3} \mathrm{He}$ atoms (which in reality would be separated by an average spacing of about $7 \times 10^{-8} \mathrm{~m}$ ), e - purer He II whose ${ }^{3} \mathrm{He}$ content falls with increasing distance $x$ from the main bath, f- isotopically purified ${ }^{4} \mathrm{He}$ drawn off to receiving vessel 


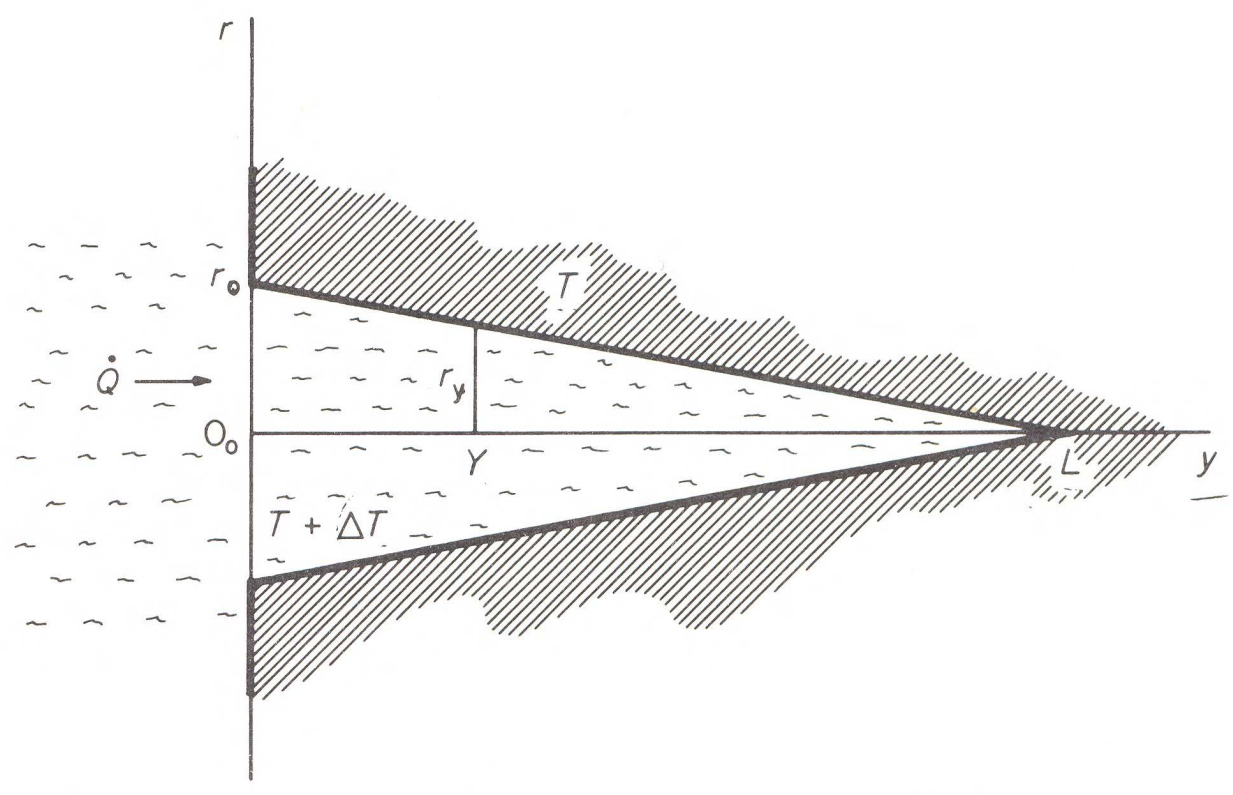

Figure 2: Conical heat exchanger for concentrating ${ }^{3} \mathrm{He}$ isotopic impurities in He II: with thermal flux $\dot{Q}$ entering the open end of the cone, causing the temperature of the liquid to rise $\Delta T$ above that of the copper walls, it can be shown (see text) that the average axial thermal flux remains the same for all positions $0<y<L$ along the axis. Consequently, the axial normal fluid velocity is also independent of position and all ${ }^{3} \mathrm{He}$ atoms should therefore be driven to the apex at $y=L$, where provision (not shown) can be made to collect them in a small subsidiary pot, behind a needle valve. 


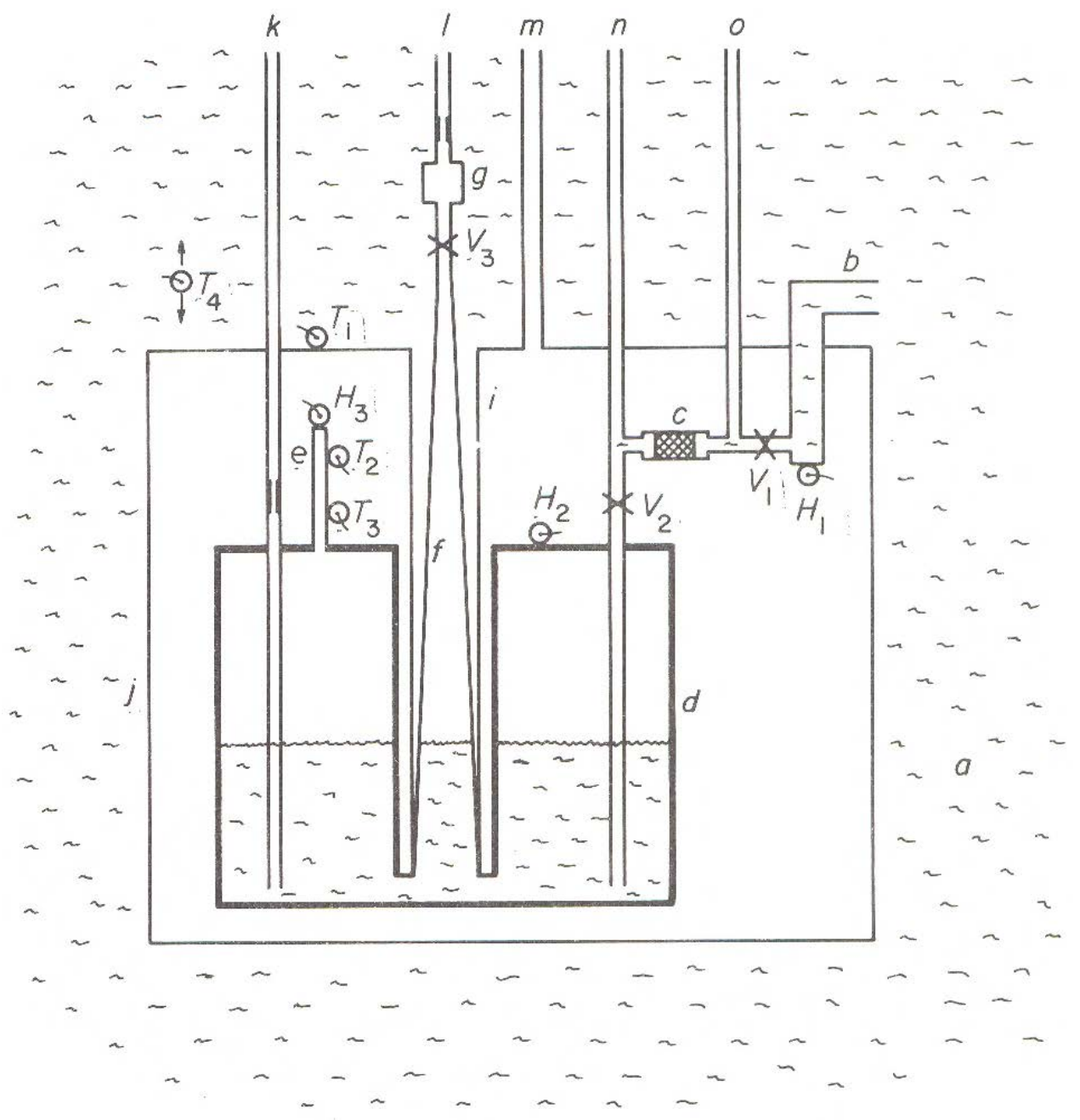

Figure 3: The lower part of the purification cryostat (diagrammatic) a - pumped bath of He II of natural isotopic composition, b - flushing tube, c - superleak, d - copper pot for receiving isotopically pure ${ }^{4} \mathrm{He}$, e - indicator to show when pot is completely filled with pure ${ }^{4} \mathrm{He}, \mathrm{f}$ - copper concentrator cone, g - small subsidiary pot for receiving the ${ }^{3} \mathrm{He}$ atoms (if any) flushed out of the main pot d, i - brass support tube, $\mathrm{j}$ - vacuum can, $\mathrm{k}$ exit tube for isotopically pure product, 1 - exit tube for concentrated sample, $\mathrm{m}$ - pumping tube for vacuum can, $\mathrm{n}$ and o - pumping tubes for superleak, $\mathrm{H}_{1}, \mathrm{H}_{2}$ and $\mathrm{H}_{3}$ - heaters, $\mathrm{T}_{1}, \mathrm{~T}_{2}, \mathrm{~T}_{3}$ and $\mathrm{T}_{4}$ - thermometers, $\mathrm{V}_{1}, \mathrm{~V}_{2}$ and $\mathrm{V}_{3}$ - needle valves. 


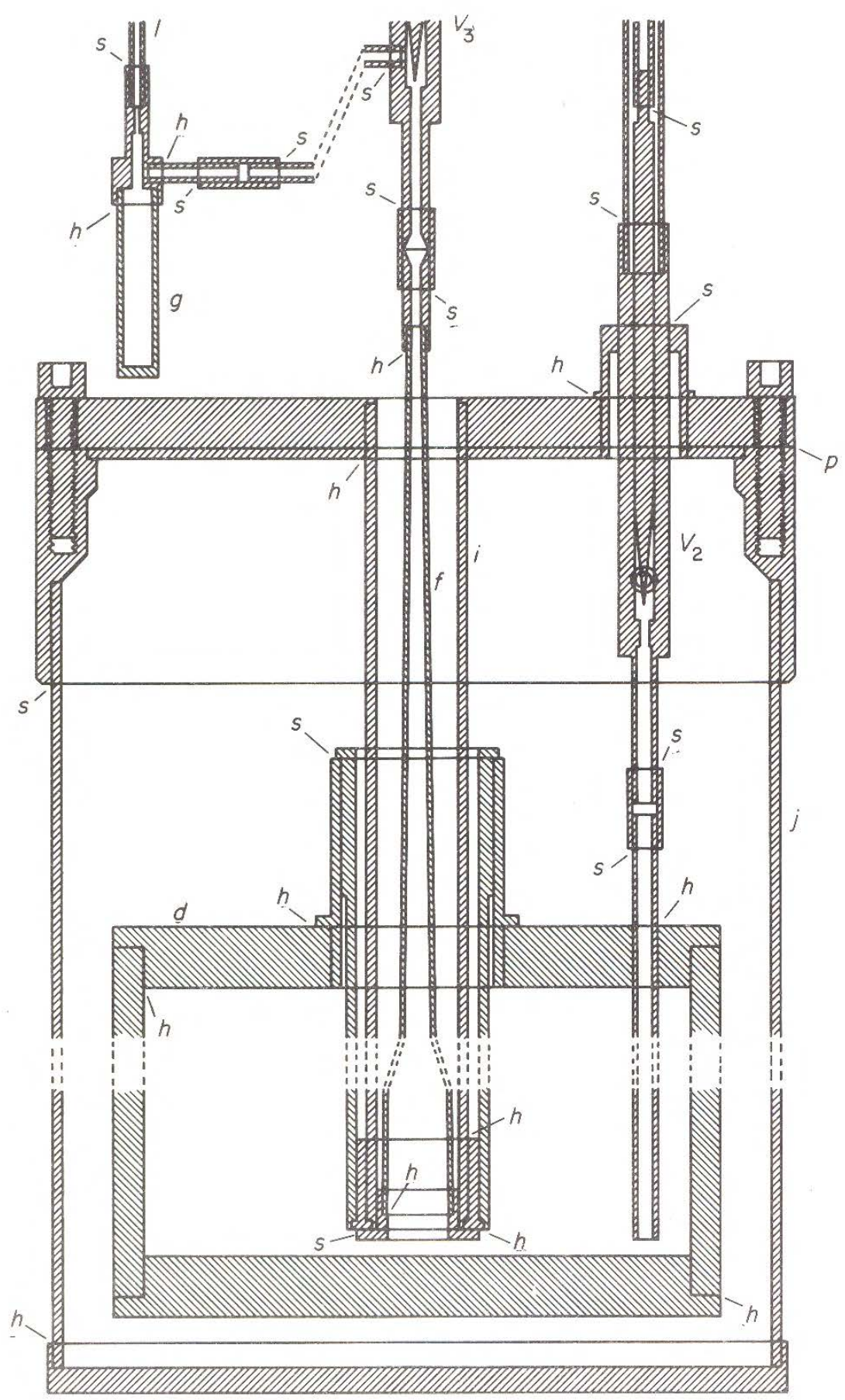

Figure 4: Vertical section of the lower part of the purification cryostat, showing the method of construction $\mathrm{h}$ - hard soldered joint, $\mathrm{p}$ - indium ' 0 ' ring, $\mathrm{s}$ - soft soldered joint, components labelled as in Fig. 3. Copper components are shaded NW-SE; brass and steel components. are shaded NE-SW. To save space, most of the main pot d has been omitted: in reality it was of height $27 \mathrm{~cm}$, od $11.5 \mathrm{~cm}$. The pipework used for admitting purified He II from the main bath is shown in Fig. 5. 


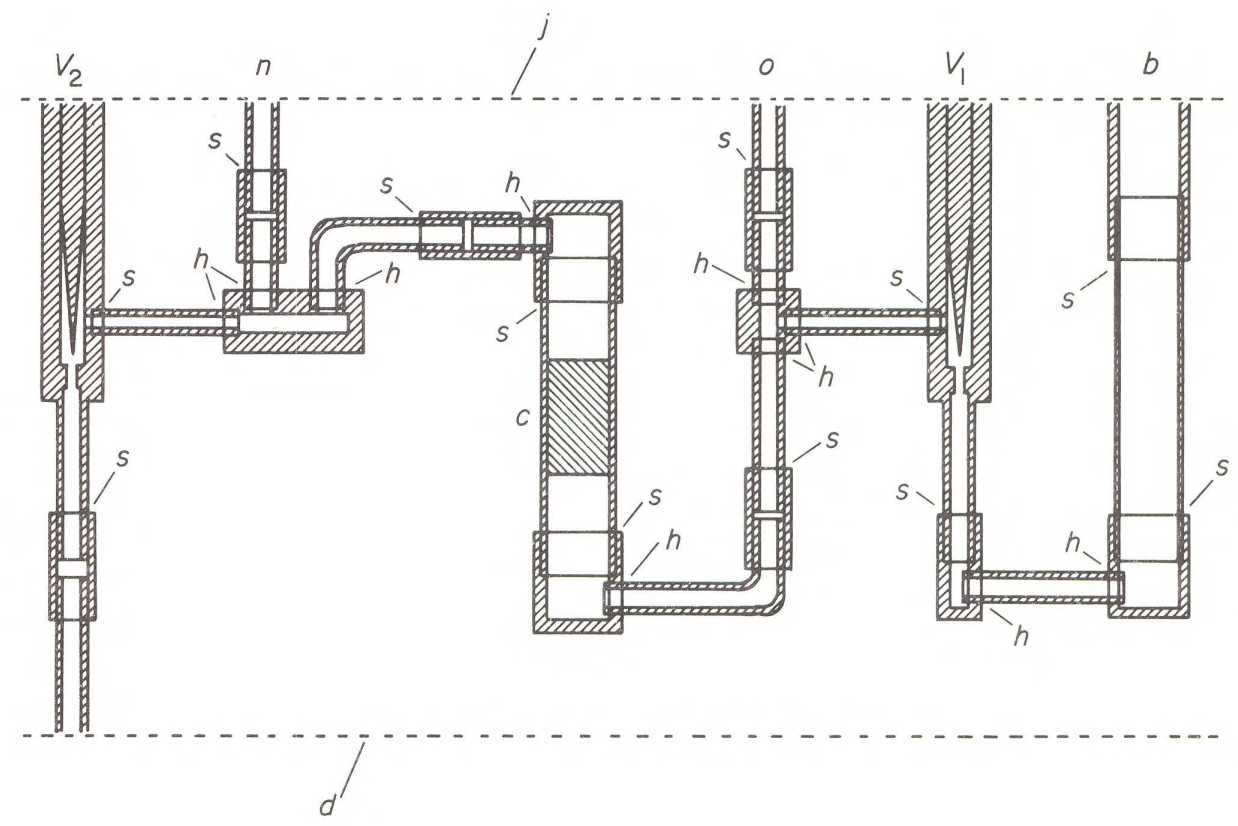

Figure 5: Pipework connecting the flushing tube, needle valves and superleak, showing the method of construction $\mathrm{h}$ - hard soldered joint, s - soft soldered joint, components labelled as in Fig. 3. The upper and lower dashed lines refer respectively to the positions of the vacuum can flange and of the top of the main pot. In the actual cryostat, the components were bent round to form an arc of circle of radius $5 \mathrm{~cm}$ and were not, as shown here for clarity, in a straight line. 


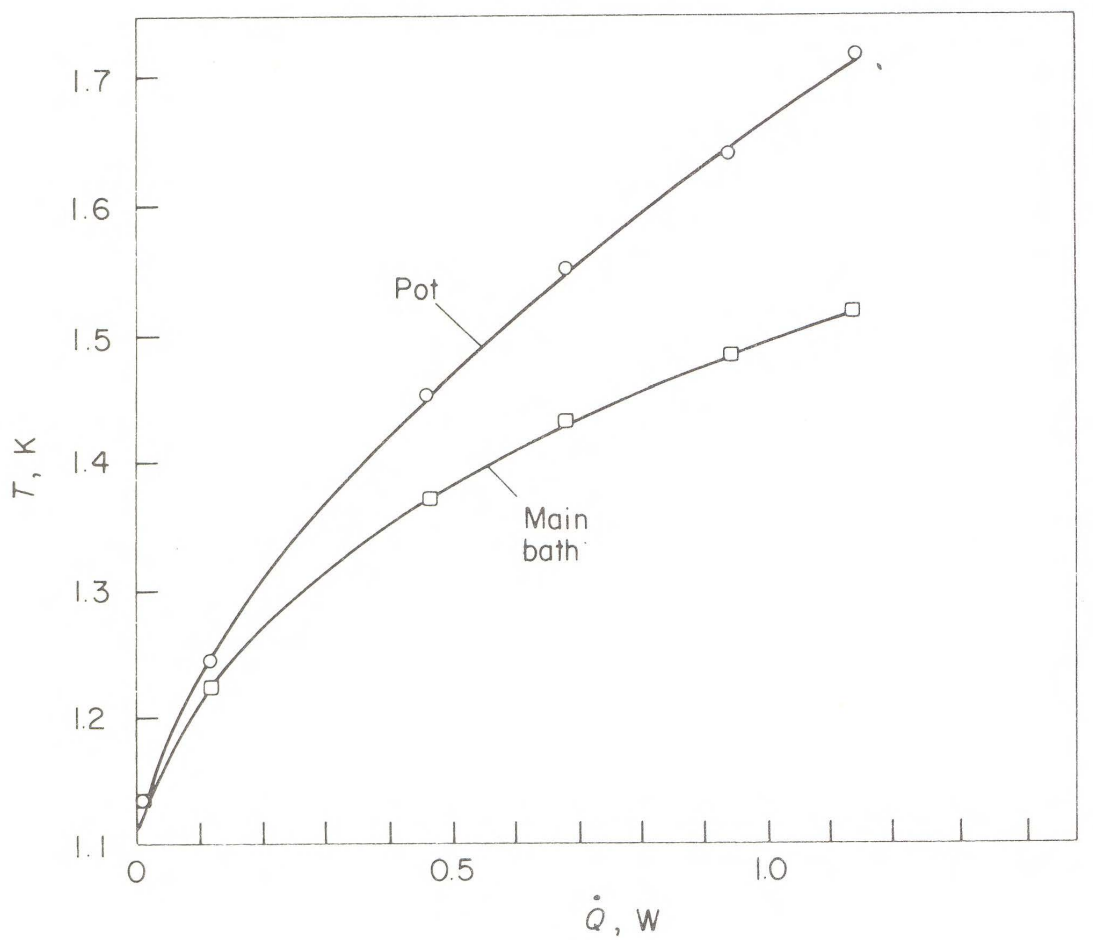

Figure 6: Characteristics of the purifier under thermal load, with the throttling valve between the dewar and helium pump fully opened. In response to a given heat input $\dot{Q}$, the temperature $T$ of the pot rises above that of the helium in the dewar, mainly because of the Kapitza temperature discontinuities between the liquid and the copper on either side of the cone. 


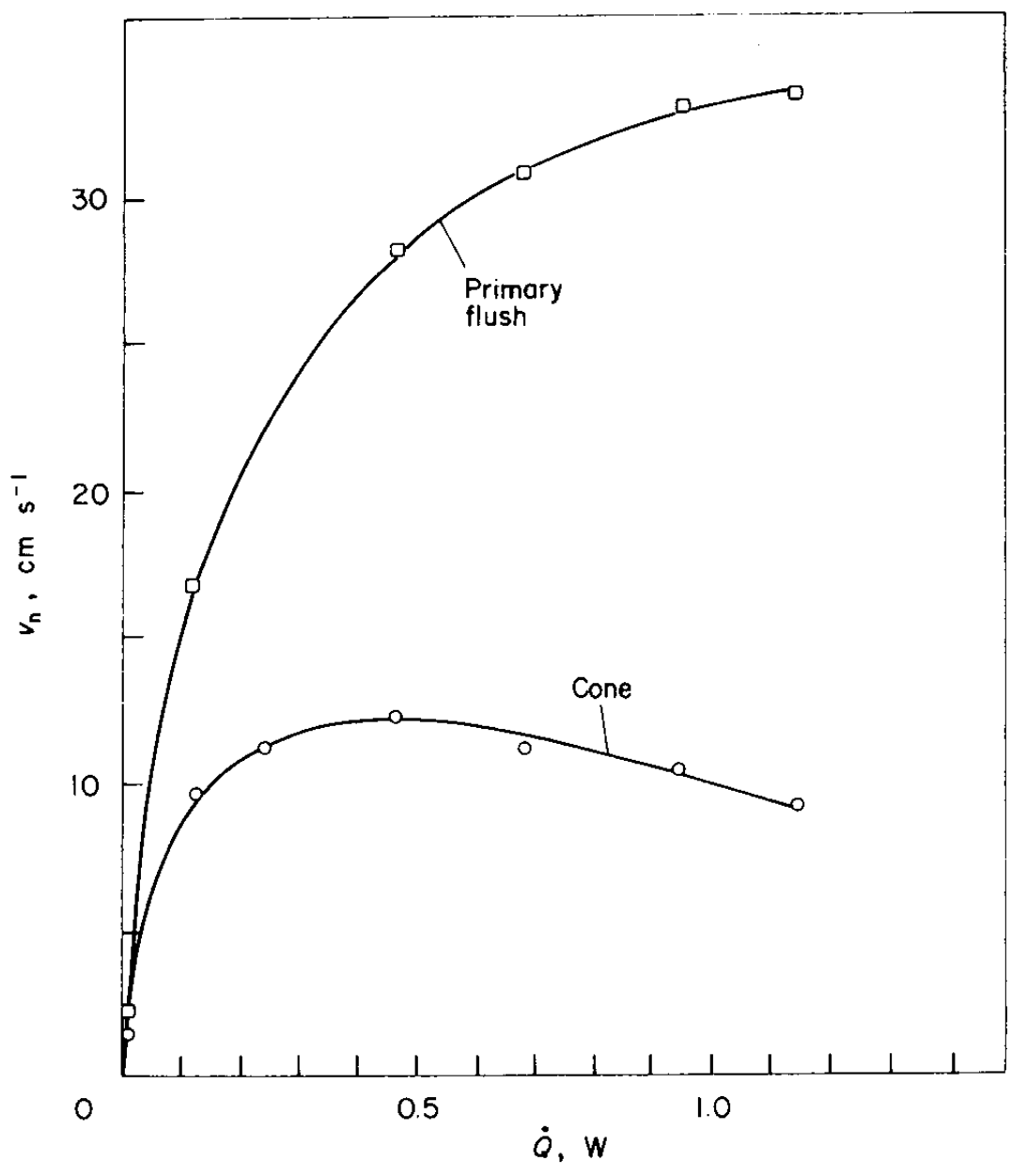

Figure 7: Normal fluid velocities $v_{n}$ calculated from (7) for the outlet of the flushing tube (primary flush) and for the inlet at the base of the cone (secondary flush) as functions of the heat $\dot{Q}$ applied respectively to the heaters $\mathrm{H}_{1}$ and $\mathrm{H}_{2}$ (see Fig. 3) 